\section{Case Reports in Ophthalmology}

\title{
Triamcinolone Acetonide-Assisted Descemetectomy for Retained Descemet Membrane
}

\author{
Ariel Tyring $^{a} \quad$ Oliver Chang $^{b}$ Hoon Jung ${ }^{a, b}$ \\ aDepartment of Ophthalmology, University of Washington, Seattle, WA, USA; \\ ${ }^{b}$ Department of Veterans Affairs Puget Sound, Seattle, WA, USA
}

\section{Keywords}

Retained Descemet membrane $\cdot$ Corneal transplant

\begin{abstract}
We present a case of decreased vision due to the presence of retained Descemet membrane (DM) following Descemet stripping automated endothelial keratoplasty (DSAEK) and penetrating keratoplasty (PKP). A 78-year-old male presented postoperatively from PKP after previous failed DSAEK with retained DM. We present a unique technique for combined surgical resection using bimanual vitrectomy and forceps excision assisted by triamcinolone acetonide for membrane visualization. Histopathological evaluation confirmed incomplete DM removal during the PKP. With surgical excision, there was an improvement in best spectaclecorrected visual acuity from $20 / 80$ to $20 / 30$.

\section{Introduction}

Retention of a Descemet membrane (DM) following keratoplasty is an uncommon complication of corneal transplant surgery. Most case reports are in the setting of penetrating keratoplasty (PKP) [1-4]; however, recent reports have demonstrated membrane retention following endothelial keratoplasty as well [5]. Visual acuity can be threatened directly by opacification of these retained membranes or secondarily by compromise of endothelial 
function, leading to graft failure. Various techniques for management have been described previously [6-9]. We describe a unique technique for surgical excision of retained DM in a case with prior sequential endothelial keratoplasty and PKP.

\section{Case Report}

A 78-year-old man with a history of two prior glaucoma drainage device procedures who had undergone Descemet stripping automated endothelial keratoplasty (DSAEK) for bullous keratopathy followed by full-thickness PKP for DSAEK failure presented with complaints of decreased vision. Visual acuity in the affected eye at initial assessment was 20/80. Anterior segment examination was notable for the presence of a retained DM with the appearance of a double anterior chamber as well as peripheral anterior synechiae with iris attachments to the graft (Fig. 1).

Prior PKP surgery had included a manual scissor excision of the DSAEK button followed by completion of a new full-thickness keratoplasty with focal anterior synechiolysis. Anterior segment optical coherence tomography imaging revealed the presence of a tissue layer consistent with retained DM in the anterior chamber (Fig. 2). Full disclosure was made to the patient along with a discussion of management options, and definitive surgical removal by anterior vitrectomy was recommended.

The patient underwent uneventful surgery with topical and subconjunctival anesthesia. The anterior chamber was accessed via 1-mm limbal incisions and the membrane was highlighted with triamcinolone acetonide. The membrane was resected from the anterior chamber using a combination of bimanual vitrectomy and forceps excision. Dense adhesions between the retained membrane, iris tissue, and corneal graft were observed intraoperatively and, after unsuccessful attempts to free these adhesions, the decision was made to leave adherent portions of retained membrane that were not in the visual axis.

Histopathology with PAS stain confirmed the incomplete DM removal from prior failed DSAEK button removal (Fig. 3a, b). The patient's postoperative course was uneventful, and 6 months postoperatively he had a spectacle-corrected visual acuity to 20/30 and acceptable intraocular pressure readings.

\section{Discussion}

Retained DM is an uncommon complication of PKP or endothelial keratoplasty. Factors that contribute to retention of DM include abnormalities of the graft-host interface such as persistent host DM, inflammatory debris, or fibrous ingrowth [10]. Material within the grafthost interface interferes with tissue adherence, leading to graft detachment and subsequent DM retention. Additionally, corneal edema facilitates DM separation, which may result in membrane detachment during trephination for repeat transplant, which is the likely cause of detachment in our patient [9].

Non-interface posterior retained membranes compromise vision when the membranes themselves become opaque from fibrous ingrowth or fibrous metaplasia of donor endothelium. Additionally, retained membranes contribute to graft failure by compromising endothelial cells and disrupting anterior chamber aqueous nutrition $[2,4]$.

Removal of retained membrane is indicated when vision or graft viability are threatened. Options include the use of neodymium-doped yttrium aluminum garnet (Nd:YAG) la- 
Case Reports in
Ophthalmology

Case Rep Ophthalmol 2018;9:227-231

DOI: 10.1159/000487706

(c) 2018 The Author(s). Published by S. Karger AG, Basel www.karger.com/cop

Tyring et al.: Triamcinolone Acetonide-Assisted Descemetectomy for Retained Descemet Membrane

ser to create an opening in the membrane within the central visual axis [8]. May et al. [9] reported a case in whom femtosecond laser was used to dissect the membrane followed by intraocular forceps removal. Nd:YAG-assisted descemetectomy was not recommended in this case due to concern for possible obstruction of glaucoma drainage devices by loose membrane scrolls. As such, surgical excision was indicated for visual restoration.

The previously described techniques for surgical DM excision include use of forceps to retrieve detached DM, intraocular scissors to resect membrane within the visual axis, and creation of a membranotomy using a 27-gauge needle and a continuous curvilinear capsulorhexis method $[2,4,6]$. In cases such as ours with dense adhesions, use of intraocular forceps and scissors alone to free DM from attachments can be cumbersome and involve substantial tissue manipulation. Bimanual vitrectomy offers the advantage of precise tissue cutting with decreased intraocular manipulation. Furthermore, the use of trypan blue stain has been suggested to highlight the DM [7]. Application of triamcinolone acetonide is by now a well-recognized method for improved intraoperative visualization of vitreous and membranes and was selected in this case to assist with visibility and to provide distinct demarcation of the retained membrane [11].

\section{Conclusion}

This case report highlights a rare, but visually significant complication of keratoplasty and describes a unique surgical technique for resection of retained DM. To our best knowledge, the use of bimanual vitrectomy assisted by triamcinolone acetonide for removal of retained DM has not been reported so far in the literature.

\section{Acknowledgments}

The work to present this case report was supported by an unrestricted grant from Research to Prevent Blindness (RPB).

\section{Statement of Ethics}

Patient consent was obtained along with institutional review board approval for publication of this case report. No personally-identifiable information was utilized.

\section{Disclosure Statement}

The authors have no conflicts of interest or proprietary interest in any of the topics or products presented in this paper. 


\section{References}

1 Lifshitz T, Oshry T, Rosenthal G: Retrocorneal membrane after penetrating keratoplasty. Ophthalmic Surg Lasers 2001;32:159-161.

2 Chen YP, Lai PC, Chen PY, Lin KK, Hsiao CH: Retained Descemet's membrane after penetrating keratoplasty. J Cataract Refract Surg 2003;29:1842-1844.

3 Thyagarajan S, Mearza AA, Falcon MG: Inadvertent retention of Descemet membrane in penetrating keratoplasty. Cornea 2006;25:748-749.

4 Choi JS, Oh JY, Wee WR: A case of corneal endothelial deterioration associated with retained Descemet's membrane after penetrating keratoplasty. Jpn J Ophthalmol 2009;53:653-655.

5 Mondloch MC, Giegengack M, Terry MA, Wilson DJ: Histologic evidence of retained fetal layer of the Descemet membrane after presumed total removal for endothelial keratoplasty: a possible cause for graft failure. Cornea 2007;26:1263-1266.

6 McVeigh K, Cornish KS, Reddy AR, Vakros G: Retained Descemet's membrane following penetrating keratoplasty for Fuchs' endothelial dystrophy: a case report of a post-operative complication. Clin Ophthalmol 2013;7:1511-1514.

7 Sinha R, Vajpayee RB, Sharma N, Titiyal JS, Tandon R: Trypan blue assisted descemetorhexis for inadvertently retained Descemet's membranes after penetrating keratoplasty. Br J Ophthalmol 2003;87:654-655.

8 Masket S, Tennen DG: Neodymium:YAG laser optical opening for retained Descemet's membrane after penetrating keratoplasty. J Cataract Refract Surg 1996;22:139-141.

9 May W, Alrashidi S, Daoud Y: Removal of retained Descemets membrane using femtosecond laser. Middle East Afr J Ophthalmol 2016;23:259-261.

10 Romaniv N, Price MO, Price FW, Mamalis N: Donor Descemet membrane detachment after endothelial keratoplasty. Cornea 2006;25:943-947.

11 Couch SM, Bakri SJ: Use of triamcinolone during vitrectomy surgery to visualize membranes and vitreous. Clin Ophthalmol 2008;2:891-896.

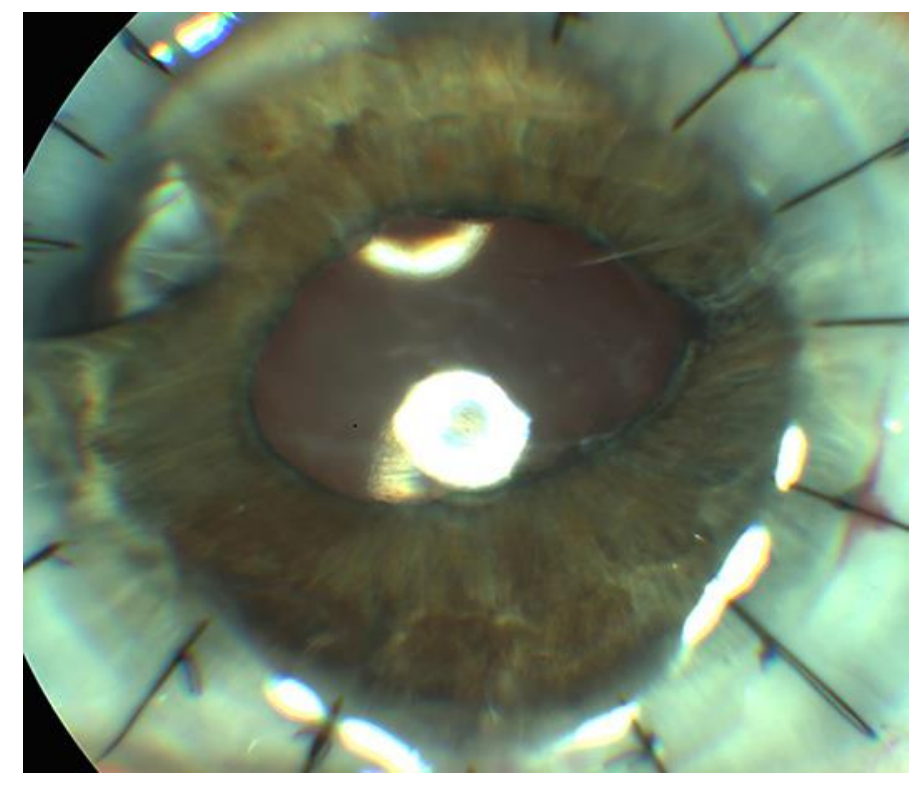

Fig. 1. Slit lamp photograph demonstrating fine Descemet membrane scrolling within the anterior chamber centrally and a triangular window defect superior and temporal in the membrane. 


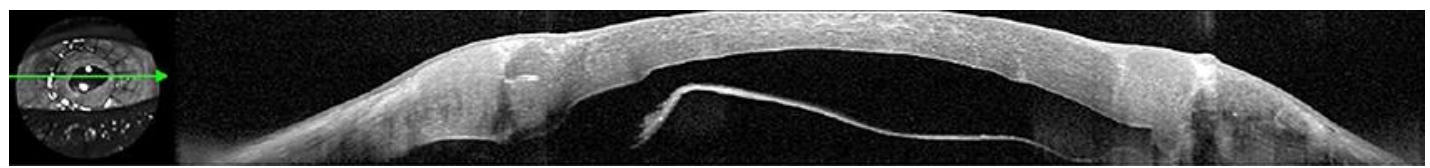

Fig. 2. Anterior segment optical coherence tomography demonstrating the retrocorneal membrane floating free within the anterior chamber.

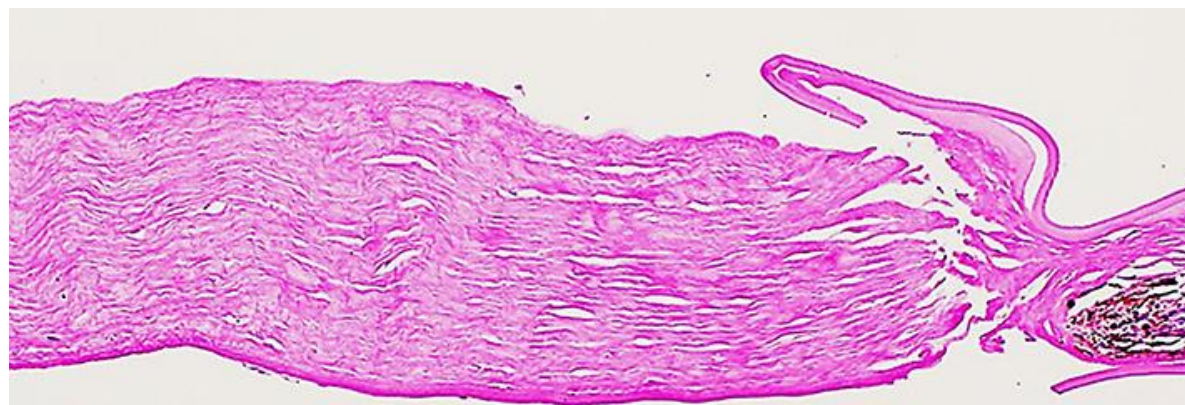

a

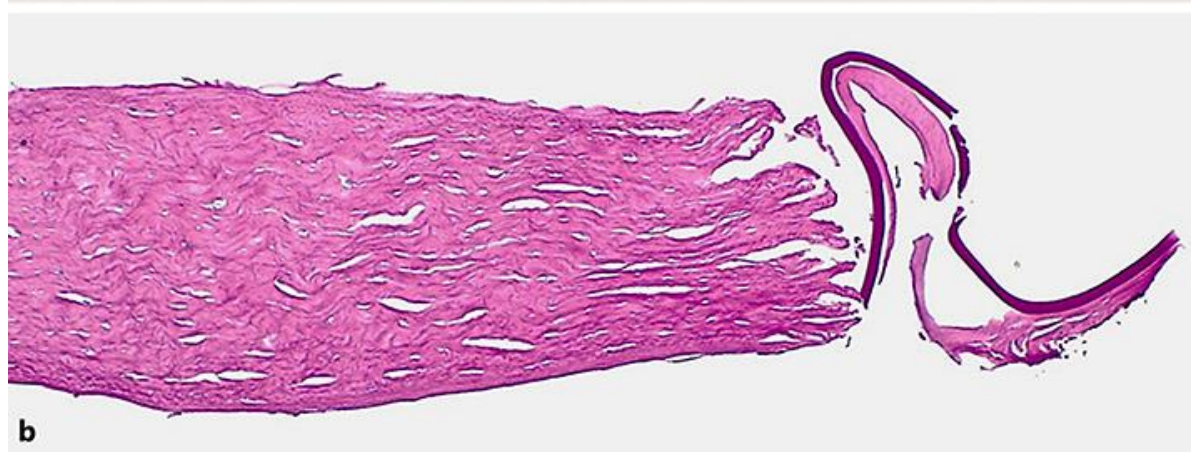

Fig. 3. Histopathology sections of the retained Descemet membrane. a Corneal stroma comprising collagen fibrils with characteristic clefts from processing artifact. $\mathrm{H} \& \mathrm{E}, \times 10$. $\mathbf{b}$ The residual peripheral Descemet membrane is highlighted with PAS stain towards the right side of the image. PAS, $\times 10$. 